the line of thought which I have endeavoured to present to you to-night. 1. Certain appendices are so formed anatomically that they of necessity give rise to trouble. 2. Interference with the drainage of the appendix is an important factor in determining inflammation. 3. The occurrence of appendicular colic may be taken as a warning that dangerous anatomical conditions exist in connexion with the appendix. 4. In all abdominal cases detailed palpation should be carefully and patiently carried out. 5. Too much emphasis must not be put on the site of pain or on superficial tenderness. Palpation should be directed towards ascertaining the actual condition of the diseased part. 6. Statistics show that from 90 to 95 per cent. of cases recover without operation and the chances against recurrence are as three to one or thereby. Therefore routine removal cannot be accepted as necessary. 7. Operate at once in any case with a history of previous attacks, also in all cases commencing with a rigor. 8. The chief difficulty lies in the early recognition of the cases in which the appendix will necrose or perforate rapidly. In this connexion suspect cases which show constitutional disturb ance with little inflammatory reaction round the cæcum but with marked tenderness on deep palpation and an ordinary degree of leucocytosis. 9. In cases which do not call for immediate operation pain may be relieved by hot or cold local applications and by a cautious and sparing use of opium or morphine or of belladonna. Lavage may give good results. 10. Medical treatment should not be relied on for more than from 24 to 48 hours if severe pain continues or an acute tenderness on deep palpation still remains. 11. Later in the disease a continuance of the local and general symptoms along with a high leucocyte count indicates the presence of pus and the necessity for operative interference.

Bibliography. - Bourget: Typhlite, Pérityphlite, Appendicite. Brown : International Clinics, vol. iv., twelfth series, 1903. Da Costa American Journal of the Medical Sciences, November, 1901. Deaver A Treatise on Appendicitis Philadelphia, 1900. Dunham: Annals of A Treatise on Appendicitis, Philadelphia, 1900 . Dunham: Annals of delphia, 1894. Hawkins : On Diseases of the Vermiform Appendix 1895. Huns : On Diseases of the Vermiforma Appendix London, 185. Hubbard : Boston Tedical and Surgical Journal, Phy Cxians Philadeiph 1899 Kelynack Pations, Pathology of the Vermiform Appendix, London, 1893. Legueu Traitement de 189. Nothnagel: Skolikoiditis und Perityperapie, Band xvii.

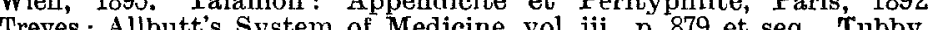

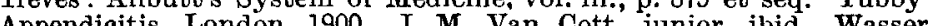

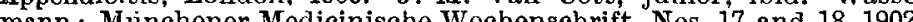
Tdinburgh.

\section{THE EARLY DIAGNOSIS OF ACUTE INTESTINAL OBSTRUCTION. ${ }^{1}$}

BY W. HALE WHITE, M.D., F.R.C.P. LOND.,

PHYSICIAN TO, AYD LECTURER ON MEDICLNE AT, GUY'S HOSPITAI,

Ir will, I think, be found that most of as look upon the early diagnosis of intestinal obstruction in a somewhat different way from that in which it was usually regarded 40 years ago. This is because our skill in diagnosis has advanced, but in saying this I am speaking of medical men generally, for it is doubtful whether even the best of us are more skilful than the most brilliant diagnosticians of half a century ago, but as the average of skill has risen we less frequently see cases in which acute intestinal obstruction has been so long overlooked that when the patient comes to a hospital the diagnosis is obvious. Then, too, the re sponsibility attached to a correct diagnosis has increased enormously in this and many other diseases of the abdomen. Before surgery could help us to any appreciable extent i mattered comparatively little whether or not the diagnosis was correct, for if the patient was suffering from acute intestinal obstruction we could, unless it was due to an external hernia, do little or nothing to relieve him. But now the responsibility of the physician has increased ten thousandfold, for if he fails to suggest that a surgeon should see a case which the necropsy shows to have been suitable for operation it is always quite likely that early surgical interference might have saved the patient's life. On the other hand, the physician may urge the surgeon to operate unnecessarily and on all grounds unnecessary operations are most undesirable.

1 Being the introductory remarks at a discussion at the Medical Society of London on March 14th, 1904
Formerly much importance was attached to the elucida. tion of the problem as to whether the obstruction was high up or low down, but Fagge pointed out in his classical paper on which most subsequent descriptions have been founded, that often the supposed points of difference in the symptoms were untrustworthy, and we now know that the symptoms are more dependent upon the cause of the obstruction than upon its situation. He classified his cases according to the cause and all the best descriptions since have followed him, as it is important for the surgeon to know the cause for which he has to search. But the result of this treatment of the subject is that very often accounts of acute intestinal obstruction fail to give sufficient prominence to the conditions for which such obstruction may be mistaken, and therefore, as the subject put before us to-night is the early diagnosis of acute intestinal obstruction, I thought the most practical way to attack it would be first to mention the conditions which I have known to be mistaken for it, especially as the best way to prevent mistakes in diagnosis is to be aware of their possibility.

It might be supposed that it would often be difficult to distinguish between severe acute cases of appendicitis nnaccompanied by general peritonitis and acute intestinal obstruction but, as a matter of fact, the difficulty only occasionally arises at the bedside and then it will, I think, be found that in most instances of confusion acute appendicitis is mistaken for intestinal obstruction but not of the most acute variety, rather than that the converse mistake is made. The following is a case in point, One Friday evening I saw with Dr. Slater-Jones a man, aged 60 years, who gave a history of severe attacks of colic, once accompanied by jaundice. On the previous Wednesday evening he was suddenly seized with a severe rigor. Dr. Slater.Jones soon arrived and found the patient extremely collapsed with a temperature of $104^{\circ} \mathrm{F}$. The next morning it was $99^{\circ}$ and normal in the evening, there was some abdominal pain but it was across the top of the abdomen, he was sick twice, and we were told that the bowels had been opened on this day. On Friday he was sick only in the morning and when $I$ saw him in the evening the abdomen was uniformly moderately distended without any dulness, tenderness, or other sign to indicate local disease. The distension made me fear we should have to open the abdomen later but as the pulse was very good, he did not look ill, his temperature was $98^{\circ}$, and he said that he felt better, we decided, as it would have been very difficult to arrange any operation that night, to leave him till the morning, believing that if he continued to improve we should have to conclude he was suffering from gall-stone colic without jaundice or from abdominal influenza. I heard early Sunday morning that he was not so well, therefore $\mathbf{M r}$. Charters J. Symonds joined us and we learnt that on Saturday there had been frequent very foul stercoraceous vomiting and that we had been misinformed about his bowels they really had not been opened, not even any flatus being passed, since Wednesday in spite of several enemata. His pulse was 110 and of good volume ; he looked well ; and his temperature was $98^{\circ}$. His abdomen was distended uniformly without any indication of local disease but we decided upon operation because of the obstruction and continuing distension. We all thought the most likely diagnosis was that there was a gall-stone impacted in his ileam but operation showed a small abscess around the appendix in which a slough had led to a perforation; the peritonitis was not. general. Here was a case in which severe and absolute constipation, distension, and stercoraceous vomiting were due to appendicitis which, on the other hand, did not give any indication of its presence and in which the initial rise of temperature only lasted 24 hours. We did not forget that an examination of the blood might have helped us but unfortunately I had not any of the necessary apparatus with me when $I$ arrived at the patient's house. In another case of doubt I have known the presence of leucocytosis correctly turn the balance in favour of an abscess connected with the appendix.

It will be noticed that the case of appendicular abscess just recorded, unlike as it was to most cases of appendicitis, did not lead to a diagnosis of acute obstruction but to one of obstruction of a more chronic kind-viz., by a gall-stoneand I think it is extremely rare for the locailised peritonitis, caused by disease of the appendix, to be mistaken for acute intestinal obstruction, but it is less rare for a mistake to be made between acute general peritonitis and intestinal obstruction and this is not surprising, for strangulation of the bowel renders possible the passage of bacteria through it 
and therefore many cases of acute obstruction quickly become complicated by acute peritonitis, hence it follows that the difficulty of diagnosis is often less the earlier the case is seen, but, on the other hand, probably the symptoms of few diseases vary so much as do those of acute peritonitis. Speaking generally, absence of pyrexia and of leucocytosis is in favour of intestinal obstruction, so also is rapid distension of the abdomen, especially if coils of intestine in which peristalsis is visible can be seen; early and urgent vomiting quickly becoming stercoraceous is in favour of obstruction; the pain is often paroxysmal in obstruction, the abdomen is not board-like or very tender, and there is no cutaneous hyperæsthesia; the patient does not lie in the usual attitude of peritonitis but may bend forward, doubled up by the pain, and the abdomen moves with respiration until later in the disease than is the case with acute peritonitis. But it is essential to bear in mind that every one of these symptoms which have been mentioned as generally indicating obstruction may be met with in peritonitis in which there may be complete absence of the passage of fæces and flatus by the bowel.

Volvulus is the form of intestinal obstruction which most resembles acute peritonitis, nor is this surprising, for owing to the large amount of bowel strangulated and the severity of the strangulation the bowel rapidly permits the passage of micro-organisms which set up peritonitis. Probably the chief distinguishing feature of volvulus is the distension of the abdomen which is, as a rule, much more extreme and rapid than with acute peritonitis and perhaps the pain is less severe and the temperature is lower in volvulus.

Acute hæmorrbagic pancreatitis is apt to be mistaken for intestinal obstruction; the form which it most nearly resembles is that due to acute strangulation of the small intestine but if the patient is suffering from acute pancreatitis the actual obstruction is not well marked, the bowels are often opened several times soon after the onset of the pain, although later they are constipated, there is no distension of the intestines, and as the case goes on the vomiting does not often become stercoraceous. Should the patient live long enough there are signs of a localised peritonitis and pancreatitis at the upper part of the abdomen, the chief being localised tenderness, a feeling of resistance, and later distension due to the formation of a localised abscess with its circumjacent peritonitis. Probably by this time the bowels have been opened again and slight pyrexia has supervened. Several cases of pancreatitis have been recorded as surviving and laparotomy has been undertaken under the impression that they were cases of intestinal obstruction, but there is no evidence that in such the operation has been of benefit; indeed, it may have turned the scale against the patient; but whether we shall become sufficiently sure of a diagnosis of acute pancreatitis to withhold exploratory laparotomy is doubtful but at any rate this is the goal to be aimed at. It need hardly be said that if a distinct abscess forms operation will be needed.

Perforation or rupture of any hollow viscus or cyst within the abdominal cavity may be mistaken for intestinal obstruction, especially strangulation of the small intestine, but the mistake is one of the least serious because immediate laparotomy is necessary for either perforation or obstruction. The difficulty is at its greatest when the previous history gives no suspicion that rupture might occur-e.g., there is nothing to lead us to suspect a gastric ulcer or an ovarian cyst. Our chief guides to correct diagnosis will be that the onset of the symptoms is more severe and sudden with per. foration even than with strangulation of the small intestine by a band and symptoms of peritonitis supervene much more early with perforation. The vomiting is more likely to be severe and later to become stercoraceous in obstruction in which the pain is almost always referred to the umbilicus and local tenderness is no guide to the seat of obstruction but not inirequently local tenderness and pain are guides to the seat of perforation. The abdomen usually becomes immobile and distended earlier in perforation than obstruction.

Thrombosis and embolism of the mesenteric vessels both lead to symptoms which may closely resemble those of acute intestinal obstruction. Fortunately the conditions are rare, for nothing but harm can follow opening the abdomen. Usually there is some obvious cause for the trouble, as heart disease which might cause embolism or some intra-abdominal trouble which might canse thrombosis. The sudden abdominal pain-referred to the umbilicus in both casesand the collapse are equally marked in vascular obstruction and intestinal obstruction, in both vomiting is early and may become stercoraceous but probably it is altogether a more striking symptom in intestinal obstruction. If a vessel corresponding to a large area of bowel is blocked abdominal distension will be earlier than in a case of acute strangulation of the small intestine but the characteristic symptom is the passage of much blood from the bowel and if the superior mesenteric artery or vein is blocked some blood may regurgitate into the stomach and be vomited. Sometimes the blocking occurs in the aorta and then if this be in such a situation that the mesenteric arteries are interfered with there will be in addition to the symptoms due to interference with the mesenteric vessels severe sudden cramp-like pain and loss of power in the legs. The diagnosis from intestinal obstruction is then of course easy. A patient recently under my care who was suffering from heart disease was suddenly taken with pain and complete loss of power in the legs, abdominal pain and collapse so severe that he died in ten hours in spite of treatment; embolism of the abdominal aorta implicating the mesenteric arteries was found. I need hardly add that if the renal arteries are implicated hæmaturia will be an aid.

Intestinal colic is a fruitful source of error and many a patient's life might have been saved if he had not been regarded as suffering from what is called "simple colic." I need not take up the time of a society such as this with the diagnosis of lead colic which I have seen mistaken for acute intestinal obstruction. I would only point out that severe vomiting is quite the exception in lead colic and that we too of ten neglect to look for lead in the urine. With regard to other forms of intestinal colic it is important to remember that very severe intestinal colic-restricting the term to pain due to some local irritation usually caused by an error of diet-is rare in adults, while, on the other hand, acute intestinal obstruction-except that due to intussusception which has its own special symptoms-is rare in children, so that the opportunities of confounding intestinal colic with acute intestinal obstruction ought to be few. It is other forms of abdominal disease-e.g., appendicitis-rather than acute intestinal obstruction which are often erroneously set down to colic. Still, the mistake does occur and it is mainly to be avoided by noting the severity of the symptoms. If the patient's abdominal pain is so severe that he-an adult-sends for a medical man who finds that there is no diarrhcea and that there is no obvious indication of any canse for the pain such as a renal calculus, lead, disease of the appendix, \&c., but the symptoms are not severe enough to indicate certainly acute intestinal obstruction, nothing should then be done except to apply warmth to the abdomen and to give an enema if there has been constipation, but no opium, as that will mask the symptoms. On a second visit a few hours later it will usually be found, if the case is one of acute intestinal obstruction, that the severity of the pain, the collapse, the state of the pulse, and the frequent urgent distressful vomiting leave no room for doubt and the abdomen should be opened at once. In the cases under discussion usually the severity and frequency of the vomiting and the state of the pulse are the first symptoms to show that the cause of the pain is acute intestinal obstruction, later the patient's expression and his collapse show this disease, in which it will be borne in mind the pain is often relieved by vomiting.

Biliary and renal colic can hardly ever be confused with acute intestinal obstruction if care is taken. Here, again, the severity of the vomiting and the collapse together with the facial expression and the feeble rapid palse met with in intestinal obstruction will prevent an error. I have known the abdominal pain in a patient who had ulcerative colitis with constipation lead to an erroneous diagnosis of intestinal obstruction.

Acute intussusception may be confused with severe diarrhoea in children but as a matter of fact the mistake can nearly always be avoided if sufficient care is taken. The detection of a tumour either in the abdomen or the rectum is, of course, conclusive and the sufferer from acute intussusception at first has bloody diarrbcea, later constipation supervenes, but he commonly at some time passes pure blood, whilst in the severe enteritis of children the motion, if blood is present at all, consists of fæces, blood, and mucus throughout and constipation does not supervene till the patient is convalescent. Even if the case of diarrhœa is so severe as soon to lead to collapse this is usually preceded by pyrexia and often in these cases there is frequent and severe vomiting, but this is not usually a striking symptom in intussusception unless severe constipation comes on early in the case. Cholera may closely resemble acute obstruction. 
There are some conditions which are met with in women which may be difficult to tell from acute obstructionnamely, the twisted pedicle of an ovarian cyst, a ruptured ovarian cyst, a ruptured extra-uterine foetation, a ruptured pyosalpinx, and hysteria. In the three cases of rupture these are really instances of perforative peritonitis and therefore all that has been said in connexion with the diagnosis of that from acute obstruction will apply here. I have seen a ruptured extra-uterine fotation mistaken for acute appendicitis, for it led to a tumour and feeling of resistance on the right side, but the slight elevation of temperature and the signs of pregnancy soon led to a correct diagnosis. In the only case of a twisted pedicle of an ovarian tumour leading to difficulty that I have met with the presence of a palpable right-sided tumour which was tender led to a diagnosis of appendicitis, which was perhaps a pardonable mistake as the patient was only 12 years old In most cases the locality of the pain and tenderness, with in some the detection of a tumour, will usually lead to a correct diagnosis from acute intestinal obstruction. I have known great care necessary to distinguish hysteria from acute peritonitis but hysteria does not simulate acute intestinal obstruction.

Lastly, in any case of severe pain in the abdomen we should think of pleurisy and pneumonia but when early in the course of either of these the symptoms are mainly abdominal they suggest peritonitis rather than obstruction. I have seen pneumonia thought to be peritonitis but never obstruction.

Having very briefly reviewed the conditions which may be mistaken for acute obstruction we pass on to the diagnosis of the kind of obstruction and here it is of fundamental importance to remember that the early symptoms of acute intestinal obstruction are caused by the strangulation of the bowel and not by its obstruction. This is illustrated by the fact that a volvulus of the large intestine gives very sudden acute and serious symptoms-because of the strangulation-while the symptoms of a complete obstruction-say, by a gall-stone-without strangulation are much less severe. Indeed, the more we think of it the less important does mere obstruction become. For example, I remember the case of a woman whose bowels had not been moved'for a month, it was thought owing to the pressure of a huge renal tumour compressing the bowel, but the tumour was really a great mass of fæces blocking the descending colon. She was not acutely ill. We do not observe acute symptoms even in spite of prolonged constipation in the cases in which the intestine is compressed by peritoneal adhesions due to the various forms of peritonitis. Think of the state of the intestines all compressed into one mass, such as we see at a post-mortem examination on a case of chronic peritonitis, yet there are no acute symptoms. The other day I saw the abdomen opened for chronic obstruction; the intestines were all matted together, the bowels had been rarely opened, and only with the greatest difficulty, but there were no symptoms of acute obstruction. The upshot of this is that to diagnose the form of obstruction we must, if the symptoms are severe and acute, say to ourselves the patient has got some form of obstruction that is likely to be associated with strangulation-e.g., a band, internal hernia, volvulus, kinking, or anything that will strangulate the bowel, and he is not suffering from compression, stricture, or impaction of contents unless these are complicated by strangulation. It illustrates my point to remind you that intussusception is severe in proportion as the bowel is strangulated. It is true that if a large number of obstructions of the small intestine are contrasted with a large number of obstructions of the large intestine the symptoms of the former will usually be much more acute than of the latter but this has nothing to do with the position of the obstruction; it is because whatever obstructs the small intestine generally at the same time strangulat 3 it. Usually speaking, there are no acute symptoms unless there is strangulation and the symptoms of this are sudden agonising pain, often paroxysmal, referred to the umbilicus, frequent, early, distressful, and severe vomiting, a rapid, feeble pulse, an ashy, pinched countenance, a normal or subnormal temperature, and collapse so severe that a pre viously healthy man may in a few hours be at death's door, hence when these exist we infer that the bowel is strangulated and it would be more accurate to diagnose such a case as acute strangulation of the bowel rather than as acute intestinal obstruction, for as already mentioned, these symptoms only enable us to diagnose strangulation of the bowel irrespective of the seat or cause of the strangulation. If there is great and rapid distension in addition to the symptoms of strangulation we infer a volvulus; if there is history of chronic obstruction-e.g., that due to malignant growth in the large intestine-the distended bowel behind the obstruction has become kinked or strangalated in some way ; there may be other evidence of intussusception; or if, as is usually the case, we decice none of these are present and there is no external hernia, then, arguing from the experience of the post-mortem room, we learn that the small intestine is almost certainly the seat of the strangulation, a view which is supforted if thus early in the case there is not much intestinal distension, for distension in such a case is partly due to obstruction of the bowel above the strangulation-but in strangulation of the small intestine there is not much of this-and partly due to paralytic distension of the strangulated bowel, but usually when the small intestine is strangulated not much of it is affected. But when we have arrived at the diagnosis of strangulation of the small intestine we cannot from the symptoms say what part of it is affected or the cause of the strangulation. Our knowledge of morbid anatomy shows that the cause of the strangulation may be one of many, such as a band of some sort, an internal hernia, \&c. Excluding external hernias internal strangulations are far the most important variety of obstruction to diagnose early, for (1) they are frequently of such a nature that the surgeon can completely relieve them; (2) unless relieved they will quickly kill ; (3) they usually come in previously healthy adults in the prime of life; and (4) there is usually no concomitant disease likely to kill the patient.

Among intestinal obstructions belonging to the entirely different class in which there is no strangulation, obstruction of the ileum by a gall-stone is one of the most acute, but here the fact that the patients are nearly always women over 50 years of age while strangulation usually occurs in men under the age of 50 years will be of help. Further, the acute symptoms already given as indicating strangulation are rarely so severe in obstruction by gall-stones with which, too, there may be a history of jaundice. As already mentioned, chronic obstruction as by a growth may suddenly give rise to very severe acute symptoms, and these may be owing to strangulation by kinking of the distended bowel above the growth but they may also be due to perforation of a distension ulcer. When, as sometimes occurs, they show themselves suddenly in a case in which the growth of the bowel has given rise to no symptoms the diagnosis is very difficult. If acute symptoms supervene upon chronic obstruction there is almost certainly a stricture of the large intestine-and strictures of this are virtually always carcinomatous-for except gall-stones the only chronic obstruction of the small intestine is that due to compression of a large extent of bowel from some form of chronic peritonitis and this compresses so much bowel that strangulation behind the compression rarely if ever occurs. Perhaps this is the best place to mention that visible mapping out of the distended bowel is a most uncertain symptom, for it may be absent in obstruction or it may be present in cases in which no obstruction is present-e.g., peritonitis-and, again, it may be impossible to tell whether it is the large or small intestine which is distended. Variation in the amount of urine passed is another symptom on which but little reliance can be placed.

I am very fully aware of the numerous shortcomings of these opening remarks which have had to be brief owing to the limited time at my disposal, but they are only intended. as a basis for discussion. Fortunately, at the bedside the difficulties are of ten less than they appear on paper.

Harley-street, $\mathbf{w}$.

\section{A CASE OF ECLAMPSIA TREATED BY THE ADMINISTRATION OF CHLOROFORM.}

\section{BY JOHN FREDERIOK JENNINGS, M.B. LOND.,} F.R C.S. ENG.,

LA'TE INTERY MIDWIFERY ASSISTANT, ST. BARTHOLOMEW'S

$$
\text { HOSPITAL. }
$$

THe patient, who was a primipara, 23 years of age, was admitted into St. Bartholomew's Hcspital at 10.45 P.M. on Sept. 10th in an unconscious condition with the history that she had been seen to fall out of a railway carriage on to the platform whilst the train was still in motion. On arrival at the hospital she was not only comatose but had no corneal

$$
\text { M } 3
$$

